\title{
Translation and validation of Food Insecurity Experience Scale (FIES)
}

\section{Roselawati Mat Ya ${ }^{1}$, Suriati Sidek ${ }^{2}$, Jamalludin Ab Rahman ${ }^{3}$, Norhasmah Sulaiman $^{4}$, Noor Atirah Yahya ${ }^{1}$, Halimatun Saadiah ${ }^{1}$, Nurul Hazirah Jaafar ${ }^{1}$ \& Wan Azdie Mohd Abu Bakar ${ }^{1^{*}}$}

${ }^{1}$ Department of Nutrition Sciences, Kulliyyah of Allied Health Sciences, International Islamic University Malaysia, Pahang, Malaysia; ${ }^{2}$ Department of Psychology, Kulliyyah of Islamic Revealed Knowledge and Human Sciences, International Islamic University Malaysia, Kuala Lumpur, Malaysia; ${ }^{3}$ Department of Community Medicine, Kulliyyah of Medicine, International Islamic University Malaysia, Pahang, Malaysia; ${ }^{4}$ Department of Nutrition and Dietetics, Faculty of Medicine and Health Sciences, Universiti Putra Malaysia, Selangor, Malaysia

\begin{abstract}
Introduction: Food insecurity has a complex and multifaceted concept and definition, thus assessing it has been an ongoing challenge for researchers, health practitioners, and policy makers. Previous studies reported inconsistent findings on the prevalence and severity of food insecurity, depending on the measuring tools used. To overcome this limitation, this study aimed to translate and validate the Food Insecurity Experience Scale (FIES) for Malaysians, which has been used as a standard measurement by Food and Agriculture Organization (FAO). Methods: Two forward and backward translations involving experts in food insecurity studies and experts in language were done, as well as the pre-test and cognitive interview stipulated in World Health Organization (WHO) translation guidelines. Content and face validity were conducted as part of the validation process. Content Validity Index (CVI) was done to analyse content validity. Results: The harmonised Malay version of FIES was produced with $1.0 \mathrm{CVI}$, which was above the 0.8 criteria. Face validity showed good understandability and clarity of FIES. Conclusion: The translated Malay version of FIES had good acceptability, as well as good face validity when tested among the target audience. Thus, a full validation study of the Malay version FIES should be done before it is widely used to measure food insecurity in the population, specifically the Malaysian population.
\end{abstract}

Keywords: food insecurity, FIES, Malay language, translation

\section{INTRODUCTION}

Food is a fundamental human right, providing nutrient for human growth and development. Food security exists when all people, at all times, have physical, social and economic access to sufficient, safe and nutritious food that meets their dietary needs and food preferences for an active and healthy life (FAO, 2008). One of the challenges the world is facing now is to ensure that populations are free from hunger and achieve food

\footnotetext{
*Corresponding author: Dr. Wan Azdie Mohd Abu Bakar

Department of Nutrition Sciences, Kulliyyah of Allied Health Sciences,

International Islamic University Malaysia, Jalan Sultan Ahmad Shah,

Bandar Indera Mahkota, 25200 Kuantan, Pahang.

Fax : +609 571 6776; E-mail address: wazdie@iium.edu.my

doi: https://doi.org/10.31246/mjn-2020-0109
} 
security, which has been stated in the Sustainable Development Goals (SDG), specifically in goal number two. However, the process of understanding food security and the ways to achieve it is still debatable and ongoing worldwide. It is a complex and multidimensional concept (Norhasmah, Zalilah \& Asnarulkhadi, 2010). However, the issue remains important since hundreds of millions of people and households are still living in poverty and unable to eat enough food, not only in developing countries but in developed countries too (FAO, IFAD, UNICEF, WFP \& WHO, 2020). Recently, there are 820 million people reportedly hungry and almost 2 billion people who experienced moderate and severe food insecurity (FAO, IFAD, UNICEF, WFP \& WHO, 2019).

Low socio-economic status and poverty have always been associated with food insecurity. This situation not only causes undernutrition, but also overnutrition. The Current Population Survey in the United States demonstrated that $34.5 \%$ households with an annual income of below $130 \%$ were food insecure (Coleman et al., 2018). Studies in developing countries showed that the prevalence of food insecurity was $31.7 \%$ in rural households in India (Nagappa et al., 2020), while the prevalence of food insecurity among adult households in Malaysia was estimated to be within $47.2 \%$ to $100.0 \%$ (Norhasmah et al., 2021). Studies among lowincome households in rural Kelantan demonstrated that $83.9 \%$ experienced some kind of food insecurity (Ihab et al., 2015). A survey conducted in Kuantan revealed that $77 \%$ of households were food insecure (Roselawati et al., 2017). However, the tools to measure food insecurity may differ from one study to another.

There is a clear need to improve the tools, understanding the phenomena and frameworks used for various intervention targets, especially the vulnerable groups of population. In relation to this issue, various food insecurity measurement tools were developed such as the Radimer/Cornell Food Insecurity Scale, the Household Food Security Survey Module (HFSSM), and Household Food Insecurity Access Scale (HFIAS). The Radimer / Cornell Food Insecurity has been adapted, modified, and validated for use in many countries including Korea (Oh \& Hong, 2003), United Kingdom (Studdert, Frongillo Jr \& Valois, 2001), Malaysia (Zalilah \& Merlin, 2001) and Iran (Mohammadi et al., 2012). The Radimer/Cornell Food Insecurity Scale, HFSSM and HFIAS use the idea of perception among people who experience food insecurity, as well as the coping strategies adopted during food insecurity measurements (Radimer et al., 1992; Bickel et al., 2000). However, the listed tools are based on the perception of people having food insecurity rather than their direct experiences and behaviours.

Thus, to have a standard measurement for cross-country comparisons, the United Nations under the FAO through the Voices of Hungry Project (VoH) had developed an instrument named the Food Insecurity Experience Scale (FIES) (Cafiero, Viviani \& Nord, 2018), which is a severity metric of food insecurity at the household or individual level, based on people's self-reported experience of YES or NO answers regarding access to adequate food (Cafiero et al., 2018). FIES was developed based on three established tools, which were the Household Food Security Survey Module (HFSSM), Household Food Insecurity Access Scale (HFIAS), and Carribean Food Security Scale (ELSCA) (Smith, Kassa \& Winters, 2017; Ballard, Kepple $\&$ Cafiero, 2013). FIES has been used by almost 153 countries through the Gallop World Poll (GWP) survey for national monitoring of Sustainable Development 
Goals (SDG2) (Cafiero et al., 2018 \& Smith et al., 2017). However, the FIES instrument has not yet been translated into the Malay language. Therefore, this study aimed to translate FIES into the Malay language and validate its content and face validities.

\section{MATERIALS AND METHODS}

\section{Food Insecurity Experience Scale (FIES)}

FIES is a self-administered questionnaire consisting of eight items to evaluate an individual's or a household's experience and behaviour towards food accessibility. Specifically, it captures difficulties in accessing food due to resource constraints based on the following domains: uncertainty/anxiety feeling, changes in food quality, and changes in food quantity. Participants were required to answer yes or no to all eight questions. A raw score of 0 was provided for negative response and 1 for affirmative response. The total FIES score was the sum of scores from all eight questions and it was then further classified into the following levels of severity: food secure (0), mild food insecurity (1-3), moderate food insecurity (4-6), and severe food insecurity (7-8) (Jones, 2017).

\section{Phase 1: Translation procedure \\ Malay translation of FIES}

Prior to this study, permission for translation had been obtained from the original authors of The Food and Agricultural Organization (FAO). The translation was conducted in accordance to the standard translation guidelines by the World Health Organization (WHO, 2019). Five different stages were followed: (i) the initial translation from English to Malay by two independent bilingual translators, which were both food insecurity experts and Malay native speakers; (ii) the synthesis of the first two translations to provide a single pre- harmonised version of FIES (BMH1); (iii) the backward translation by two independent bilingual translators who were blinded from the original English version and who were both experts in English Literature and Linguistic; (iv) an expert committee review to compare the backward translations with the original FIES and consent on a harmonised Malay version of FIES (BMH2) [Note: The expert committee included food security experts and a representative from FAO]; and (v) the pre-test and cognitive interviewing of the 'BMH2' version on 20 participants to ensure that the adapted version retained its equivalent in the adapted situation and concluded with the FIES-Malay version, the final version of Malay FIES. The flow of translation is outlined in Figure 1.

\section{Phase 2: Validation procedure \\ Content and face validation of Malay version of FIES}

The initial validation of the Malay version of FIES was completed in two steps. Content validation, which aimed to assess the relevance of all eight FIES items, was conducted on eight experts from the fields of food security, nutrition, and psychology. Experts were required to evaluate each item on two dimensions (i.e., representativeness and clarity) using a four-point Likert scale, whereby 1 indicated not representative/ not clear, 2 indicated major correction needed to be representative/clear, 3 meant minor revision needed to be representative/clear, and 4 which meant representative/clear. Extra space was provided for further comments.

Face validation testing, which aimed to assess the comprehensibility of the translated items, was conducted on 40 target participants. The determination of sample size for face validity relied on the examples from previous studies due to a lack of theory. The study site involved both urban and rural areas in 
Stage I: Forward translation

- Two experts from food insecurity field independently translated the FIES from English version into target language, which was Bahasa Malaysia, and culturally adapted it to produce FIES (BM1) and FIES (BM2) versions.

I

\section{Stage II: Synthesis}

- $\quad$ FIES (BM1) and FIES (BM2) versions were compared through discussion with the experts to resolve any discrepancies and produce the pre-harmonised version of FIES (BMH1)

Stage III: Backward translation and review of translation

- Two language translators who were blinded from the original version independently translated the pre-harmonised FIES (BMH1) from the Malay language into English version producing FIES (E1) and FIES (E2).

- $\quad$ FIES (E1) and FIES (E2) were compared, through discussion with the translators to produce harmonised English version of FIES (EH). Next, EH version was compared to its original version.

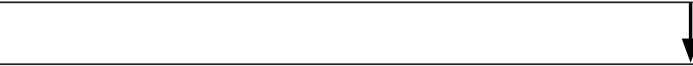

Stage IV: Expert committee review

- The experts reviewed all the reports, comments and resolved any discrepancies to produce harmonised BM version of FIES (BMH2).

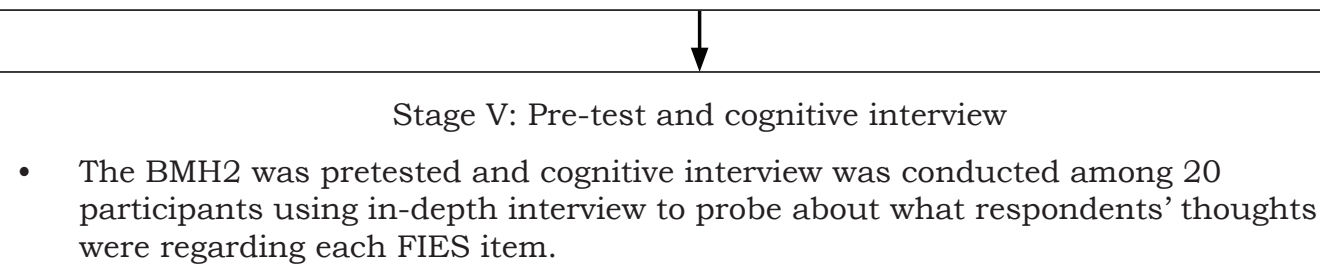

Stage V: Pre-test and cognitive interview

- The BMH2 was pretested and cognitive interview was conducted among 20 participants using in-depth interview to probe about what respondents' thoughts were regarding each FIES item.

Figure 1. Flow chart of the translation and cross-cultural adaptation of FIES

Kuantan, Pahang. The list of urban and rural areas in Kuantan was obtained from Majlis Perbandaran Kuantan $(M P K)$. The selection of the study sites, both rural and urban, was randomised but the selection of participants was based on purposive sampling. Inclusion criteria included married women of reproductive age between 19 and 49 years old. Although the age range of reproductive women in Malaysia is between 15-49 years old, those aged 1517 years were excluded as they were still schooling. Women were chosen because they are responsible for food production, purchasing and preparation, and are often the key person for household food security (Kardooni et al., 2014). Those who were lactating and pregnant were excluded. Participants were asked to 
rate the Malay version of FIES on two dimensions (i.e., understandability and clarity) using a four-point Likert scale ranging from "item not understandable/ not clear" to "item understandable/very clear".

\section{Ethical approval}

Ethical approval was obtained from the International Islamic University Malaysia Research Committee (IREC) (Ref: IIUM/504/14/11/2/REC 2019131). Consent from the participants was obtained prior to the participants answering the survey.

\section{Data analysis}

Data entry and statistical analysis were performed using IBM SPSS Statistics (Version 22, Armonk, NY, IBM Corp, Statistical Package for the Social Sciences, USA). To obtain scale level content validity index (S-CVI) of each item and content validity index (I-CVI), the scores of 3 and 4 were recategorised as 1 (representative / clear; relevant) and scores of 1 and 2 as 0 (not representative/ clear; not relevant). The I-CVI expresses the proportion of the agreement of the representativeness and relevancy of the item, which is between 0 and 1 . To obtain I-CVI, the number of experts who rated the item as 3 and 4 were counted and divided by the number of experts. The S-CVI/Ave was calculated by taking the sum of the I-CVIs divided by the total number of items (Yusoff, 2019). For face validity index (FVI) analysis, the scores from 40 participants were recategorised as 1 for clear and understandable (scores 3 and 4), and 0 for not clear and not understandable (scores 1 and 2). The universal FVI was calculated by averaging the values for clarity and comprehension, and was computed by calculating the scale average of the universal value.

\section{RESULTS}

\section{Phase 1: Translation}

In the preliminary phase of the translated version, the panels suggested modification of the sentences to suit the Malaysian culture wherever possible, but at the same time, maintaining the meaning of the translated version as the original version. Commonly, literal translation does not work for all questions. For example, the phrase 'lack of money or other resources' was confusing in Angola and it was better understood as 'lack of means' (Ballard et al., 2013). In the present study, 'other resources' was translated into "sumber-sumber lain". Most of the participants during cognitive interview session, especially urban participants, did not understand that it referred to other resources to acquire foods such as fishing, farming, transfer of food from family members or government etc. (Ballard et al., 2013). Thus, the final version was "sumber-sumber lain untuk mendapatkan makanan".

Moreover, the word 'households' refers to the people living together. The FAO expert suggested "orang-orang" to represent households, which is a phrase commonly used in Indonesia, but brings a different meaning in Malaysia. The expert panel, as well as participants then agreed with the term "ahli-ahli dalam isi rumah". In question 5, the phrase was "ate less than you thought you should because of a lack of money or other resources?". This question enquired about eating less than what the participants considered they should, even if they did not skip a meal because they did not have money or other resources. The pre-final translation was "makan kurang daripada apa yang anda fikir sepatutnya". Some of the participants understood this question was asking about eating less than they normally ate. 
But a few participants reported that the word "fikir" was confusing. They thought that the word "fikir" was referring to the changing of their mind from taking food they wanted to eat to another food which was cheaper. Thus, the final version was "makan kurang daripada apa yang sepatutnya anda makan".

Similarly, the sentence "went without eating" in question number 8 "Was there a time when you or others in your household went without eating for a whole day because of a lack of money or other resources?" cannot be literally translated into "pergi tanpa makan", which was not the intended meaning. The appropriate sentence should be "tidak makan". The linguistic challenges faced in adapting FIES into Malaysia's diverse culture are summarised in Table 1.

\section{Phase 2: Validation}

\section{Content validation by experts}

The content validation of FIES required no major corrections. The average CVI calculated was 1.00 , which was clearly above the 0.80 criteria. The experts evaluated the comprehensiveness of the entire measure and there were no suggestion of addition or deletion of any items. However, one of the experts suggested to change the FIES into a statement form instead of a question form, and to switch the position between items 4 and 5 according to its level of severity. However, we maintained the format as in the original form and the position of the level of severity was only determined after construct validation using Rasch analysis.

Face validation by target audience Among 40 participants, 33 participants $(82.5 \%)$ rated 4 for understandability and 35 participants $(87.5 \%)$ rated 4 for clarity, suggesting that the FIES item was easily understood and clear. However, FIES needed minor amendments in terms of clarity of certain terms as described in the linguistic adaptation phase.

\section{DISCUSSION}

Assessing food insecurity has evolved from capturing individual perceptions to individual lived experiences (Radimer et al., 1992; Fronggillo et al., 2013). One of the instruments to assess these experiences is through the FIES questionnaire, which is a standardised tool developed by FAO and has been used since 2014 . The FIES questionnaire has been translated into 200 languages in order to be used in many countries (Frongillo et al., 2017), so that comparison of food insecurity across countries can be made possible. Studies from other countries including the Sub Saharan Africa $(\mathrm{Na}$ et al., 2019; Sadiddin et al., 2019) Latin America and Caribbean (Smith et al., 2017) United States, United Kingdom, Australia, New Zealand, East and South Asia (Jones, 2017) have found that the FIES is relevant for the assessment of food insecurity experiences). This study aimed to translate and validate the FIES into the Malay language and is the first to report the translation and validation of FIES questionnaire in Malaysia.

The translated FIES-Malay version was linguistically valid when it was applied. Based on the findings in this study, potential threat to validity related to language translation was reduced. In this study, the content equivalence and semantic equivalence were checked during backward and forward phases to ensure that the consistency of meaning in the translated version was similar to the original version. The translation and adaptation processes implemented in this study conformed to those used in a previous study related to FIES (Ballard et al., 2013). According to Maneesriwongul \& Dixon (2004), content, context, conceptual, semantic, and technical equivalence evaluations are needed for 


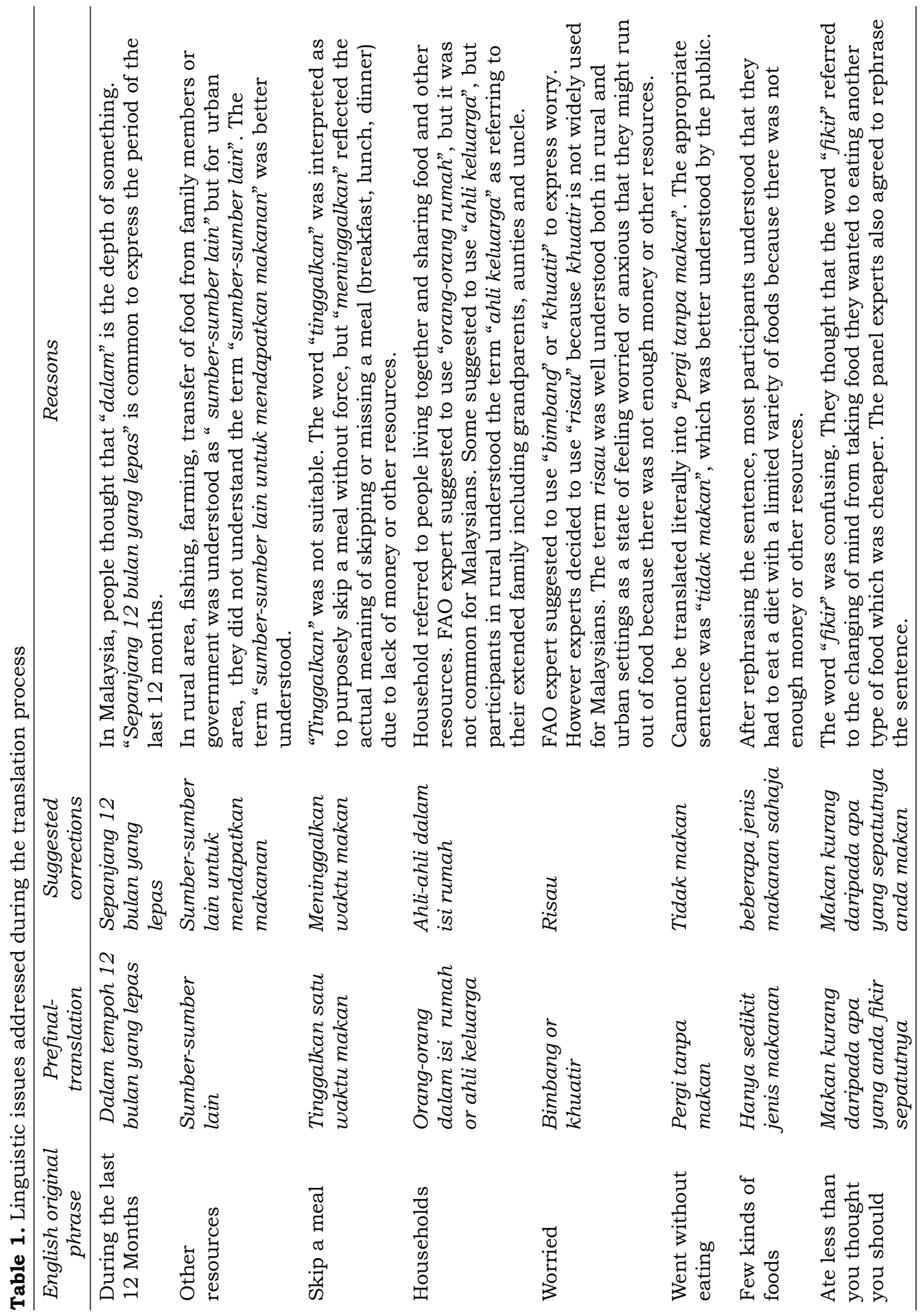




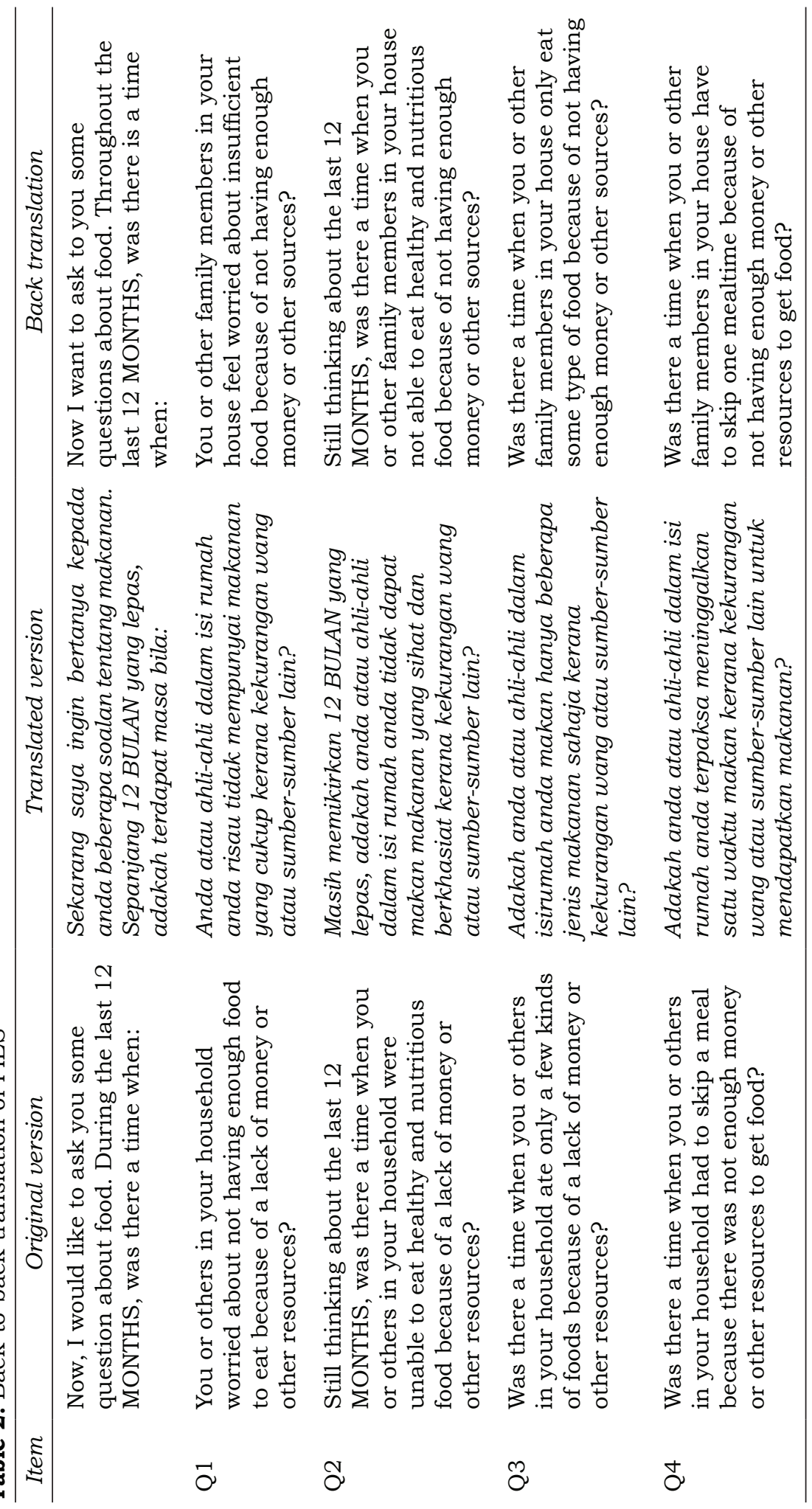




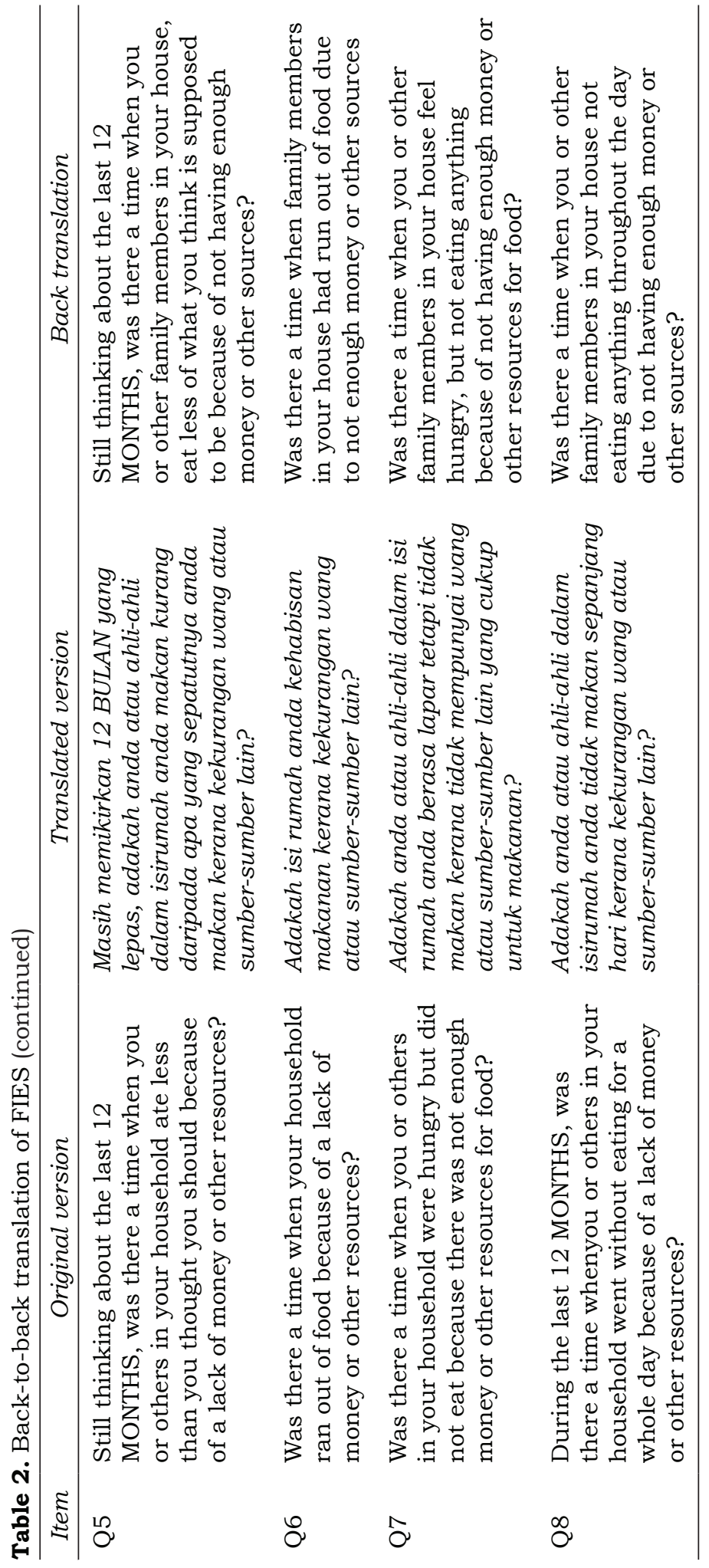


a new instrument to be used in a new setting.

In this study, the CVI scale obtained a score of 1.0, which was considered as excellent, indicating that the content of the Malay version of FIES was well adapted into the local context. The FVI scores of $82.5 \%$ and $87.5 \%$ reported in this study indicated that the original FIES had been translated into understandable and clear sentences, easily readable to all 40 targeted participants.

Based on our study, commonly, literal translation does not work because the accuracy of the result of FIES is highly dependent on the words and terminologies used in the question. Each of the FIES questions had an intended meaning with the aim of detecting experiences and behaviours associated with increased severity. The meaning and terms used for the questions must be clearly understood by the respondents to give informative answers. For example, the phrase 'healthy and nutritious' was understood differently in urban and rural populations. The rural people interpreted "makanan sihat dan berkhasiat" as eating fruits and vegetables, while the urban people understood as food that gives energy. The phrase 'went without eating' or "tidak makan sepanjang hari" could be misunderstood as fasting. Fasting is a religious Muslim practice of abstaining from taking food from dawn until nightfall. This justified the importance of backward, forward, and cognitive interview phases to ensure semantic and technical equivalence. Previous studies conducted in Angola, Ethiopia, Malawi, and Niger documented some of the language barriers in adapting FIES (Ballard et al., 2013), which were similar to the present study.

The limitation of this study was that due to different cultures, language barrier among participants occurred during the translation process, which led to the misinterpretation of terms. Although the selection of study sites for both rural and urban was randomised, but the selection of participants was based on purposive sampling, which was not the best method of sampling for the purpose of representativeness. However, this was the first study that had thoroughly translated and validated the content and face validities of FIES for the Malaysian population.

\section{CONCLUSION}

The Malay version of FIES had been translated properly, with positive expert review ratings, as well as good face validity by target audience. Thus, a full validation study of the Malay version of FIES, especially its construct validity, needs to be done before it is widely used to measure food insecurity in the Malaysian population.

\section{Acknowledgement}

This research was supported by the Fundamental Research Grant Scheme (FRGS 16-054-0553).

\section{Author's contributions}

RMY, conducted data collection, ran the analysis and wrote the manuscript; SS, JAB, NS, provided advice on data analysis, interpretation of the results, and reviewed the manuscript; NAY, HS; helped in data collection; NHJ; provided advice on data analysis, interpretation of the results, and reviewed the manuscript; WAMAB, principal investigator, conceptualised, designed the study, and reviewed the manuscript.

\section{Conflict of interest}

There is no conflict of interest to declare.

\section{References}

Ballard TJ, Kepple AW \& Cafiero C (2013). The food insecurity experience scale: developing a global standard for monitoring hunger worldwide. Technical Paper. Food and Agriculture Organization of the United Nations, Rome. From: http://www.fao.org/economic/ess/essfs/voices/en/ [Retrieved September 5 2019]

Bickel G, Nord M, Price C, Hamilton W \& Cook $\mathrm{J}$ (2000). Guide to measuring household food security. US Department of Agriculture, Food and Nutrition Service, Alexandria VA. 
Cafiero C, Viviani S \& Nord M (2018). Food security measurement in a global context: The Food Insecurity Experience Scale. Measurement 116:146-152.

Coleman-Jensen A, Rabbitt MP, Gregory CA \& Anita Singh A (2018). Statistical supplement to household food security in the United States in 2017, AP-079. U.S. Department of Agriculture, Economic Research Service, Washington DC.

FAO (2008). The state of food insecurity in the world. Food and Agriculture Organization of the United Nations.

FAO, IFAD, UNICEF, WFP \& WHO (2019). The state of food security and nutrition in the world 2019. Safeguarding against economic slowdowns and downturns. Food and Agriculture Organization of the United Nations, Rome.

FAO, IFAD, UNICEF, WFP and WHO (2020). The state of food security and nutrition in the world 2020. Transforming food systems for affordable healthy diets. Food and Agriculture Organization of the United Nations, Rome.

Frongillo EA, Nguyen HT, Smith MD \& ColemanJensen A (2017). Food insecurity is associated with subjective well-being among individuals from 138 countries in the 2014 Gallup World Poll. J Nutr 147(4):680-687.

Ihab AN, Rohana AJ, Wan Manan WM, Wan Suriati WN, Zalilah MS \& Mohamed Rusli A (2015). Assessment of Food Insecurity and Nutritional Outcomes in Bachok, Kelantan. J Nutr Food Sci 5(3): 1 .

Jones AD (2017). Food insecurity and mental health status: A global analysis of 149 countries. Am J Prev Med 53(2):264-273.

Kardooni R, Fatimah K, Siti Rohani Y \& Siti Hajar Y (2014). Traditional knowledge of orang asli on forests in Peninsular Malaysia. Indian $J$ Tradit Knowl 13(2):283-291.

Maneesriwongul W \& Dixon JK (2004). Instrument translation process: a methods review. J Adv Nurs 48(2): 175-186.

Mohammadi F, Omidvar N, Houshiar-Rad A, Khoshfetrat MR, Abdollahi M \& Mehrabi Y (2012). Validity of an adapted Household Food Insecurity Access Scale in urban households in Iran. Public Health Nutr 15(1):149-157.

Na M, Miller M, Ballard T, Mitchell DC, Hung YW \& Melgar-Quiñonez H (2019). Does social support modify the relationship between food insecurity and poor mental health? Evidence from thirtynine sub-Saharan African countries. Public Health Nutr 22(5):874-881.
Nagappa B, Rehman T, Marimuthu Y, Priyan S, Sarveswaran G \& Kumar S G (2020). Prevalence of food insecurity at household level and its associated factors in rural Puducherry: A cross-sectional study. Indian $J$ Community Med: official publication of Indian Association of Preventive \& Social Medicine 45(3):303.

Norhasmah S, Yeatman H, Russell J \& Law LS (2021). A food insecurity systematic review: Experience from Malaysia. Nutrients 13(3):945.

Norhasmah S, Zalilah MS \& Asnarulkhadi AS (2010). Food security: concepts and definitions. $J$ Community Health 16(2):2-9.

Oh SY \& Hong MJ (2003). Food insecurity is associated with dietary intake and body size of Korean children from low-income families in urban areas. Eur J Clin Nutr 57(12):1598.

Radimer KL, Olson CM, Greene JC, Campbell CC \& Habicht JP (1992). Understanding hunger and developing indicators to assess it in women and children. J Nutr Educ 24(1):36S-44S.

Roselawati MY, Wan Azdie MAB, Aflah A, Jamaludin AR \& Zalilah MS (2017). Food security status and childhood obesity in Kuantan Pahang. Int $J$ Allied Health Sci 1(2):56-71.

Sadiddin A, Cattaneo A, Cirillo M \& Miller M (2019). Food insecurity as a determinant of international migration: evidence from SubSaharan Africa. Food Secur 11:515-530.

Smith MD, Kassa W \& Winters P (2017). Assessing food insecurity in Latin America and the Caribbean using FAO's Food Insecurity Experience Scale. Food Policy 71:48-61.

Studdert LJ, Frongillo Jr EA \& Valois P (2001). Household food insecurity was prevalent in Java during Indonesia's economic crisis. $J$ Nutr 131(10):2685-2691.

WHO (2019). Process of translation and adaptation of instruments. Management of Substance Abuse. World Health Organization.https:// www.mhinnovation.net/sites / default / files / files / WHO\%20Guidelines \%200n $\% 20$ Translation $\% 20$ and $\% 20$ Adaptation $\% 20$ of $\% 20$ Instruments.docx [Retrieved September 5 2019].

Yusoff MSB (2019). ABC of content validation and content validity index calculation. Education in Medicine Journal 11(2):49-54.

Zalilah MS \& Merlin A (2001). Assesment of food insecurity among low income households in Kuala Lumpur using the Radimer/Cornell food insecurity instrument- a validation study. Mal J Nutr 7(1\&2):15-32 